Volume 9, No.1, January - February 2020

International Journal of Advanced Trends in Computer Science and Engineering

Available Online at http://www.warse.org/IJATCSE/static/pdf/file/ijatcse113912020.pdf

https://doi.org/10.30534/ijatcse/2020/113912020

\title{
Performance improvement model for Airlines connectivity system using Network Science
}

\author{
Shanmuk Srinivas Amiripalli ${ }^{1}$, Vishnu Vardhan Reddy Kollu ${ }^{2}$, B.J. Jaidhan ${ }^{3}$, L Srinivasa Chakravarthi ${ }^{4}$, \\ Vachavaya Asish Raju ${ }^{5}$ \\ 1,2,3,4,5 Department of Computer Science and Engineering, \\ GIT, GITAM University, \\ Visakhapatnam, AP, India. \\ shanmuk39@gmail.com, vishnuvardhan1709@gmail.com
}

\begin{abstract}
In recent years analytics plays an important role in understanding and increasing profits of any business. There are different types of analytic models available in market. Some of them are descriptive analytics, predictive analytics, prescriptive analytics, big data analytics etc. In this paper two different airline systems are selected and Graph analytics were applied. In initial process AirAsia and SpiceJet datasets are converted into graphs $G_{\text {air }}\left(V_{1}, E 1\right)$ and $G_{\text {spice }}\left(V_{2}, E 2\right)$. Graph analytics were applied on the above graphs and found that one i.e., $G_{\text {spice }}$ is weakly connected over $G_{\text {air }}$. A model was proposed to increase the connectivity of $G_{\text {spice }}$ so that the performance of $G_{\text {spice }}$ can be increased. So that $G_{\text {spice }}$ can increase the business by adding trips in an incremental order from one trip to 15 trips. Finally, we compared the above airline companies with the proposed model generated results and it was observed that proposed model is giving a feasible solution to $\mathrm{G}_{\text {spice }}$ by adding trips in an incremental order.
\end{abstract}

Key words: Graph analytics, TGO topology, topology optimization, NetworkX, python

\section{INTRODUCTION}

In solving many engineering applications, the best way is to represent problem in the form of diagram or a graph. In this research connectivity of two airline systems are analyzed theoretically by using network science parameters. Connectivity places an important role in increasing services to customers and also increases profits to the company. This is one of the oldest techniques inherited from Konigsberg bridge problem. We are making a new attempt to solve this problem using graph theory [1], [2].

Topology plays an important role in modern wireless technologies like WSN, adhoc networks, cyber-physical system and IOT [3]. We have basic topologies like bus, ring, star, mesh, tree, etc., which are not fulfilling all the requirements of the modern engineering problems. In this paper we are studying different models like Random network model, BARABASI-ALBERT model, Hierarchical model
[4],[5],[6]. Paper was divided into three parts, in first part introduction and literature survey was discussed followed by proposed methodology, finally results are discussed in detail.

\section{RELATED WORK}

[7] author has explained about Network science methods are applied to SCN model for growth and subsequently analyze various topological features, such as robustness attributable to highly complex and interconnected nature .The presented paper provides a comprehensive review of the methodologies adopted in literature for modeling the topology and robustness of SCNs, topological features of the real world SCNs , limitations of existing network growth models to realistically represent the observed topological characteristics and a novel perspective is proposed to impersonate the SCN topologies reported in studies, through fitness based generative network models.

[8] author has given importance of dynamic scenarios in Animals, humans, and multi-robot systems as they operate in dynamic environments they have the ability to respond to changing circumstances is paramount. An effective response requires suitable information transfer to agents this totally depend upon the interaction network. For analyzing the collective response consider an archetypal model of distributed decision-making and study the capacity of the system to follow a driving signal for varying topologies and system sizes. Experiments with a swarm of robots reveal a nontrivial relationship between frequency of the driving signal and optimal network topology. Slow- changing confusion increases with the degree of the interaction network, but the opposite is true for the response to fast-changing ones. These results are reaching implications for the design and understanding of distributed systems a dynamic rewiring of the interaction network is essential to effective collective operations at different time scales.

[9] Here the author has reviewed that complex networks have an important part in science as it mainly tells about our cultural background, perceive human interactions and consume media. The main motive behind this paper is to define the signature of a novel's story based on the topological analysis of its social network of characters, for this the author 
has built an automated tool that analyses the dialogs in novels, identifies characters and computes their relationships in time-dependent manner in order to assess the network's evolution over the course of the story.

Complex networks are characterized by highly heterogeneous distributions of links in the presence of key properties such as robustness. Several correlation measures are taken to define the characterized structure of the nets. These measures are computed for a number of real networks and analytically estimated for some simple standard models. It shows that how real networks are clustered in a well-defined domain of the entropy noise space. By using simulated annealing optimization, we suggest that constraints are actually operated on the possible complex networks [10], [11].

\section{PROPOSED TGO METHODOLOGY}

Initially air Asia and spice Jet datasets are converted into graphs, Gair(V1, E1) and Gspice(V2,E2) respectively and considered as inputs [12]. We analyse them using standard network science parameters like (n, d, E, D, K, C,T,1) which are in table 1. Detail flow of the model was explained in figure1.After analyzing them we will find out the less efficient network. If Gspice is less efficient than Gair, we will store Gair values into temporary variables and consider each edge Eiin Gair which is not Gspice and check the network with standard network science parameter. If it has better performance than previous network, consider the edge Ei otherwise donot consider it. Similarly, if Gairis less efficient than Gspice, we will store Gspice values into temporary variables and consider each edge Ei in Gspice which is not Gairand check the network with standard network science parameter. If it has better performance than previous network, consider the edge Eiotherwise donot consider it. Finally show the output for in incremental order [13], [14],[15].

\begin{tabular}{|l|l|}
\hline \multicolumn{2}{|c|}{ Table 1: } \\
Notations used in the proposed algorithms \\
\hline Symbol & \multicolumn{1}{c|}{ Description } \\
\hline Gair & airAsia network graph \\
\hline Gspice & spiceJet network graph \\
\hline V1 & Nodes of airAsia \\
\hline E1 & Edges of airAsia \\
\hline V2 & Nodes of spiceJet \\
\hline E2 & Edges of spiceJet \\
\hline $\mathrm{n}$ & Number of nodes \\
\hline $\mathrm{d}$ & diameter \\
\hline E & Edges \\
\hline D & Density \\
\hline K & Average degree \\
\hline C & Average clustering coefficient \\
\hline T & Transitivity \\
\hline l & Average shortest path length \\
\hline
\end{tabular}

\subsection{Flowchart for TGO Methodology}

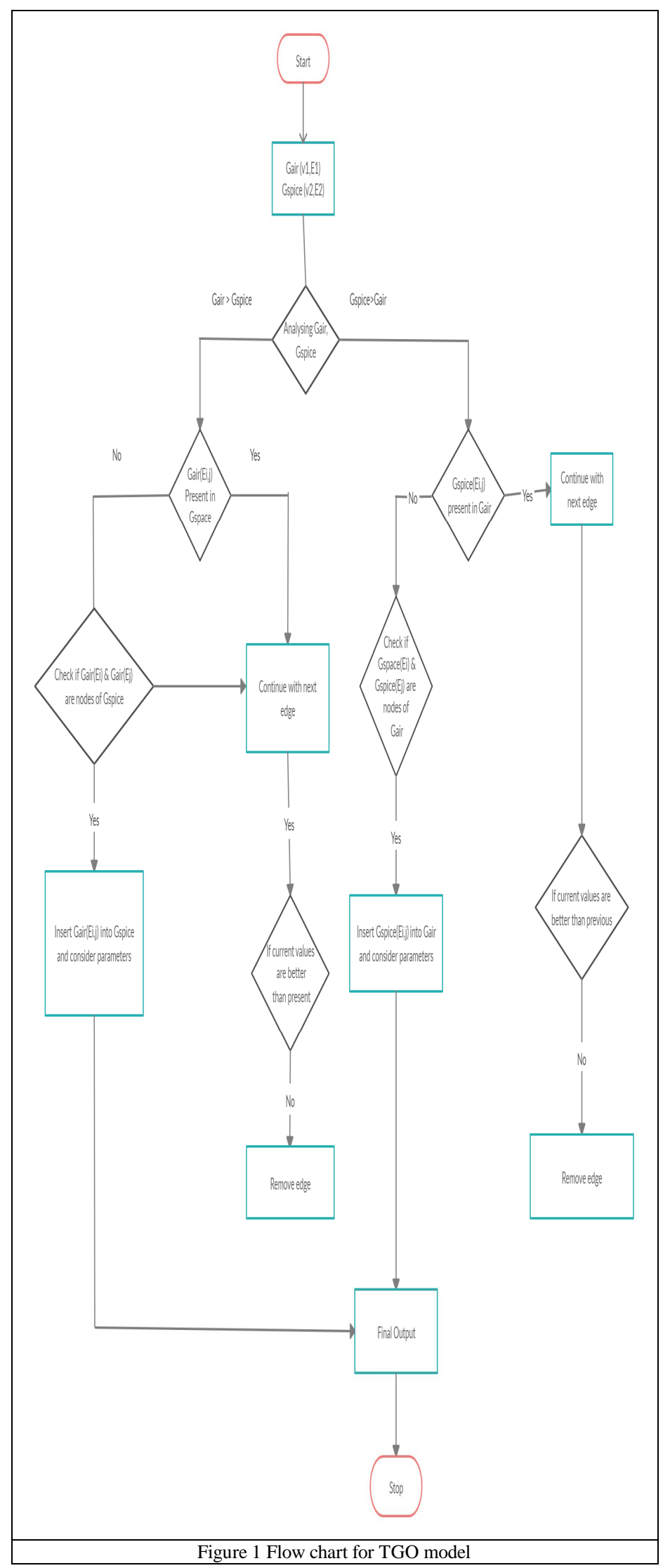




\subsection{Algorithm for TGO Methodology}

Input: $\mathrm{G}_{\text {air }}, \mathrm{G}_{\text {spice }}$

Output: $G_{\text {spice' }}$

\section{Start}

2. Let us consider $G_{\text {air }}\left(V_{1}, E_{1}\right)$ and $G_{\text {spice }}\left(V_{2}, E_{2}\right)$ as inputs

3. Analyzing $G_{a i r}, G_{\text {spice }}$ by using standard network science parameters like (n, $d, E, D, K, C, T, l)$

4. Identifying less efficient graph from $G_{\text {air }}$ and $G_{\text {spice }}$

5. Improving the performance of less efficient graph

6. If $G_{\text {spice }}$ is less efficient than $G_{\text {air }}$ :

Consider the factors of air Asia and store them in temporary variables

for each edge $G_{\text {air }}\left(V_{i}, E_{i}\right)$ in $G_{\text {air }}$ :

if $G_{\text {air }}\left(V_{i}, E_{i}\right)$ not in $G_{\text {spice }}$ and $V_{i}$ nodes present in $G_{\text {spice: }}$

insertEi into Gspice and consider the parameters,

(n, d,E,D, K,C,T,l)

consider flag $=0$

if current parameters values are much efficient than values stored in temporary variables:

update temporary variables values with current factor values

update flag $=1$

if flag $==1$ :

after $E_{i}$ print the efficiency

else:

remove $E_{i}$ from $G_{\text {spice }}$

else:

Continue with next edge

7. Else If $G_{\text {air }}$ is less efficient than $G_{\text {spice: }}$ :

Consider the factors of SpiceJet and store them in temporary variables

for each edge $G_{\text {spice }}\left(V_{i}, E_{i}\right)$ in $G_{\text {spice }}$ :

if $G_{\text {spice }}\left(V_{i}, E_{i}\right)$ not in $G_{a i} r$ and $V_{i}$ nodes present in $G_{a i r}$ :

insert $E_{i}$ into $G_{\text {air }}$ and consider the parameters

,(n,d,E,D,K,C,T, )

consider flag $=0$

if current parameters values are much efficient than values stored in temporary variables:

update temporary variables values with current factor values

update flag $=1$

if flag $==1$ :

after $E_{i}$ print the efficiency

else:

remove $E_{i}$ from $G_{\text {air }}$

else:

Continue with next edge

8. Finally show output of the improved Graph

9. Stop

\section{RESULTS AND DISCUSSION}

In figure 2. a) Same number of nodes (n) are considered and edges (E) are compared with previous airasia, spice Jet.
Results of spice Jet is low so our algorithm slowly increases the performance of spice jet, at the end spice jet has good number of edges for better performance. In fig b) Diameter (d) is decreasing and similarly average degree $(\mathrm{K})$ of a network is increasing which proves the closeness and connectivity of locations is improving. In fig c) average clustering(C), transitivity (T) and density (D) of spice jet are increasing by adding edges to a network which indicates number of locations associated with respective location is increasing when compared to previous network. In fig d) the average shortest path length (l) is decreasing which shows that pathlenth for traveling from one location to another location is low. Finally we can observe detail comparison was in the below table 2 .

\section{Table 2:Results Generated by Air Asia and Spice Jet}

\begin{tabular}{|l|l|l|l|l|l|l|l|}
\hline Parameter & $\begin{array}{l}\text { Spice } \\
\text { Jet }\end{array}$ & $\begin{array}{l}\text { Air } \\
\text { Asia }\end{array}$ & $\begin{array}{l}\text { Spice } \\
\text { Jet } \\
\text { adding } \\
\mathbf{3} \\
\text { edges }\end{array}$ & $\begin{array}{l}\text { Spice } \\
\text { Jet } \\
\text { adding } \\
\mathbf{5} \\
\text { edges }\end{array}$ & $\begin{array}{l}\text { Spice } \\
\text { Jet } \\
\text { adding } \\
\mathbf{8} \\
\text { edges }\end{array}$ & $\begin{array}{l}\text { Spice } \\
\text { Jet } \\
\text { adding } \\
\mathbf{1 3} \\
\text { edges }\end{array}$ & $\begin{array}{l}\text { Spice } \\
\text { Jet } \\
\text { adding } \\
\mathbf{1 5} \\
\text { edges }\end{array}$ \\
\hline Nodes & 21 & 21 & 21 & 21 & 21 & 21 & 21 \\
\hline Edges & 41 & 50 & 44 & 46 & 50 & 54 & 56 \\
\hline Diameter & 4 & 3 & 4 & 4 & 3 & 3 & 3 \\
\hline Avg Degree & 3.9 & 4.76 & 4.190 & 4.381 & 4.761 & 5.14 & 5.33 \\
& & & 5 & 0 & 9 & & \\
\hline Transitivity & 0.35 & 0.31 & 0.379 & 0.410 & 0.476 & 0.496 & 0.484 \\
& 5 & 3 & 1 & 9 & 4 & 1 & 5 \\
\hline $\begin{array}{l}\text { Avg } \\
\text { Clustering }\end{array}$ & 0.52 & 0.55 & 0.516 & 0.532 & 0.569 & 0.623 & 0.669 \\
\hline Density & 0.19 & 0.23 & 0.209 & 0.219 & 0.238 & 0.257 & 0.267 \\
& 5 & 8 & 5 & & & 1 & \\
\hline $\begin{array}{l}\text { Avg shortest } \\
\text { Path length }\end{array}$ & 2.09 & 1.85 & 2.03 & 2.009 & 1.96 & 1.928 & 1.880 \\
& & 7 & & & & 5 & 9 \\
\hline
\end{tabular}

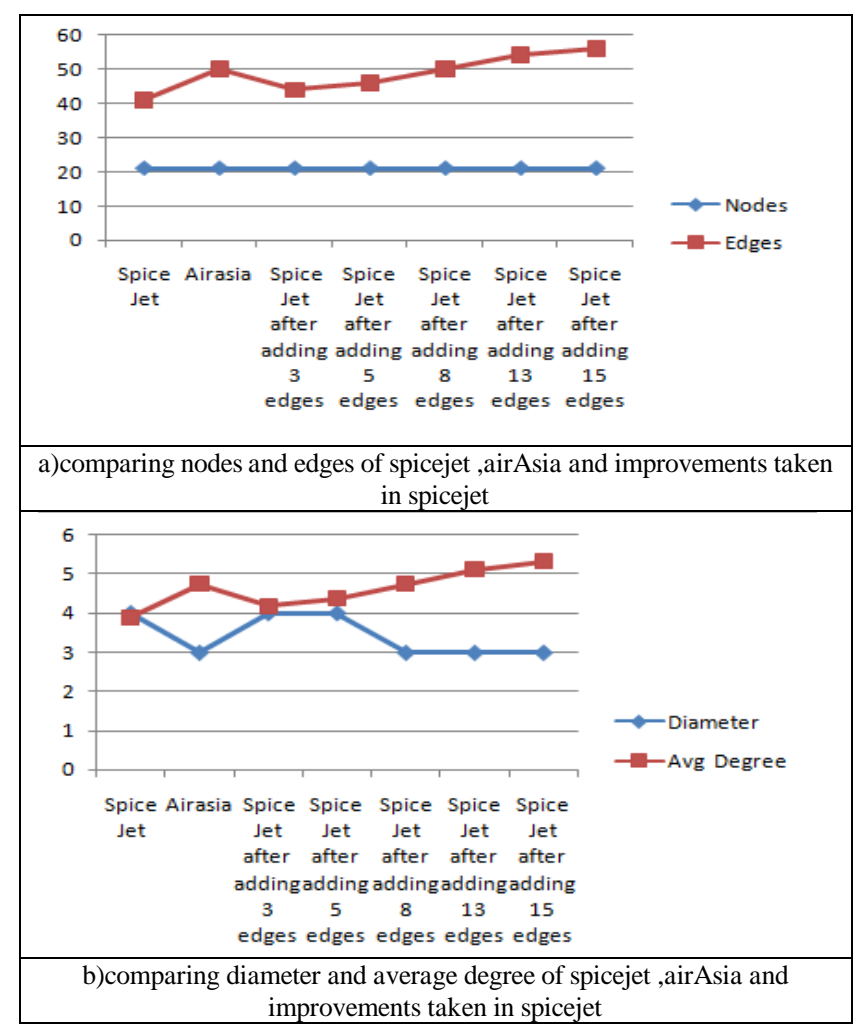




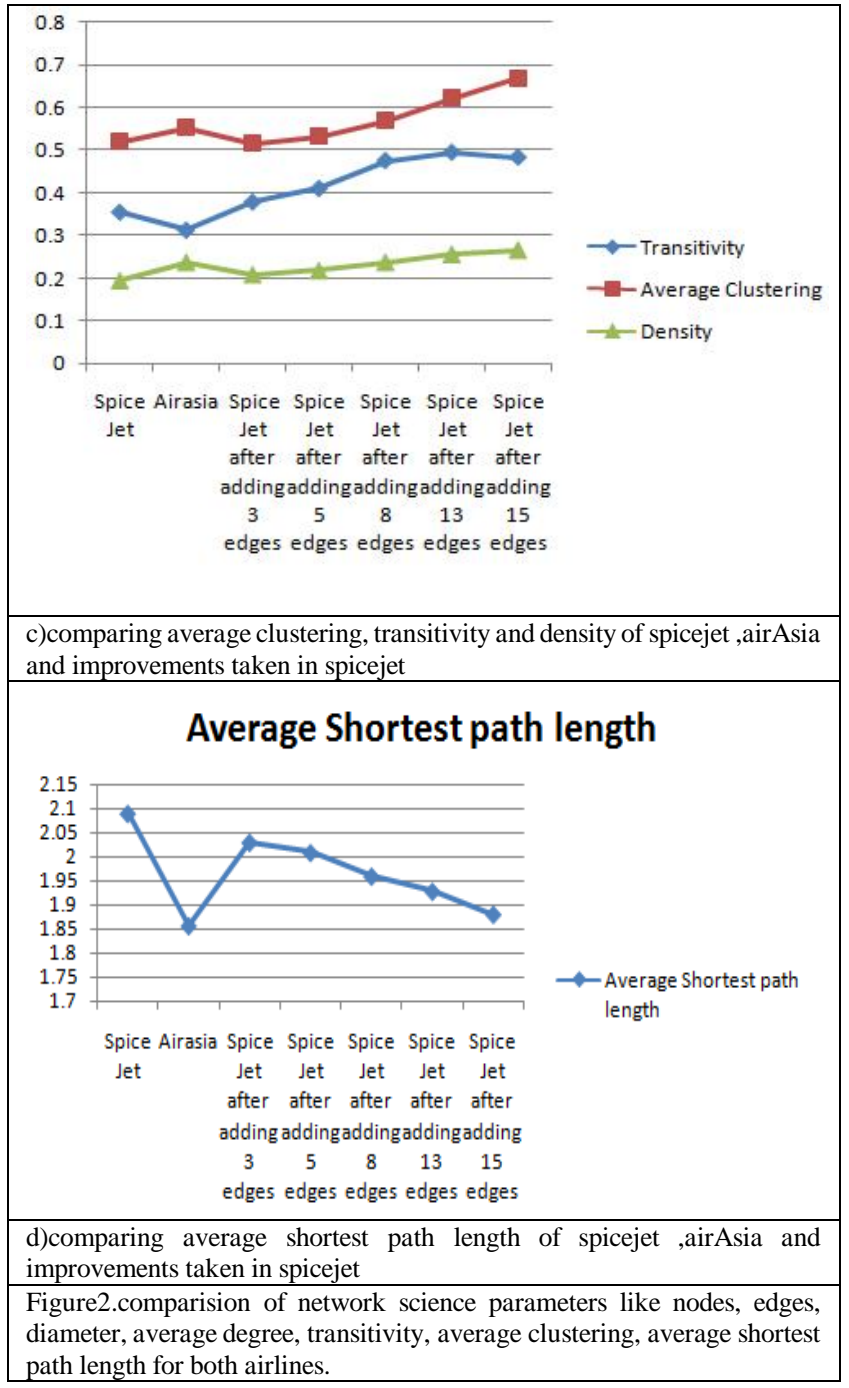

\section{CONCLUSION}

Graph analytics is one of the important techniques used for solving complex problems. In this research a complex problem will be converted into networks, for these networks standard graph theory and network science techniques was applied to get a feasible solution. In this research parameters like node, edge, and diameter, average degree, density, transitivity, average clustering, average shortest path length are used for analyzing spiceJet and airAsia. After performing graph analytics with above parameters it was observed that airAsia is well connected over spiceJet. To enhance the performance of the spiceJet a model was proposed by adding edges in an incremental order. Finally, the proposed model was compared with airAsia and spiceJet networks and it was observed that performance of proposed model shows a greater impact for giving a feasible solution to the weak connected network.

\section{Acknowledgments}

The authors would like to express their gratitude for the support offered by the Department of Computer Science Engineering, GIT, and GITAM University.

\section{REFERENCES}

[1] S.S. Amiripalli and V. Bobba, "An Optimal TGO Topology Method for a Scalable and Survivable Network in IOT Communication Technology," Wireless Pers Commun, vol. 107, no. 2, pp. 1019-1040, Jul. 2019. https://doi.org/10.1007/s11277-019-06315-Z

[2] P. C. S. Rao, P. K. Jana, and H. Banka, "A particle swarm optimization based energy efficient cluster head selection algorithm for wireless sensor networks," Wireless Netw, vol. 23, no. 7, pp. 2005-2020, Oct. 2017.

[3] S. S. Amiripalli and V. Bobba, "Impact of trimet graph optimization topology on scalable networks," IFS, vol. 36, no. 3, pp. 2431-2442, Mar. 2019.

[4] S. S. Amiripalli, V. Bobba, "Research on network design and analysis of TGO topology". International Journal of Networking and Virtual Organisations, 19(1), pp. 72-86. 2018.

[5] S. S. Amiripalli, A. K. Kumar, and B. Tulasi, "Introduction to TRIMET along with its properties and scope," presented at the Progress in Applied Mathematics in Science and Engineering Proceedings, Bali, Indonesia, 2016, p. 020032.

[6] S. S. Amiripalli, V. Bobba, "Trimet Graph Optimization (TGO) based methodology for Scalability and Survivability in Wireless Networks". International Journal of Advanced Trends in Computer Science and Engineering, 8(6), pp. 3454-3464. 2019.

https://doi.org/10.30534/ijatcse/2019/121862019

[7] S. S. Amiripalli, V. Bobba, "A Fibonacci based TGO methodology for survivability in ZigBee topologies". INTERNATIONAL JOURNAL OF SCIENTIFIC \& TECHNOLOGY RESEARCH, 9(2), pp. 878-881. 2020.

[8] Perera. Sunun. Michael Bell. and Michiel Bliemer. "Network Science annroach to Modelling Emergence and Tonological Robustness of Sunnlv Networks: A Review and Perspective." arXiv preprint arXiv:1803.09913 (2018).

[9] Mateo. David. et al. "Ontimal network tonologv for resnonsive collective behavior." Science advances 5.4 (2019): eaau0999.

[10] Waumans. Michaël C.. Thibaut Nicodème. and Hugues Bersini. "Tonologv analvsis of social networks extracted from literature." PloS one 10.6 (2015).

[11] Solé. Ricard V.. and Sergi Valverde. "Information theorv of comnlex networks: on evolution and architectural constraints." Commlex networks. Springer, Berlin, Heidelberg, 2004. 189-207.

[12] M. Al-Fares, A. Loukissas, and A. Vahdat, "A Scalable, Commodity Data Center Network Architecture, "in ACM SIGCOMM'08, Seattle, Washington, USA, 2018, pp. 1-12.

[13] M. Pal, P. Sahu, and S. Jaiswal, "LevelTree: A New Scalable Data Center Networks Topology," in 2018 International Conference on Advances in Computing, Communication Control and Networking (ICACCCN), Greater Noida (UP), India, 2018, pp. 482-486.

[14] D. Mateo, N. Horsevad, V. Hassani, M. Chamanbaz, and R. Bouffanais, "Optimal network topology for responsive collective behavior," Sci. Adv., vol. 5, no. 4, p. eaau0999, Apr. 2019.

https://doi.org/10.1126/sciadv.aau0999

[15] Sanam. Nagendram, P. Sai Anil, E. V. S. Pavan, V. Amarendra, "Performance Evaluation of Wide Area Network using Cisco Packet Tracer". INTERNATIONAL JOURNAL OF SCIENTIFIC \& TECHNOLOGY RESEARCH, 9(2), pp. 878-881. 2020. 